Review

\title{
Hydrogel-Based Platforms for the Regeneration of Osteochondral Tissue and Intervertebral Disc
}

\author{
Vincenzo Guarino *, Antonio Gloria, Maria Grazia Raucci and Luigi Ambrosio * \\ Institute of Composite and Biomedical Materials, National Research Council of Italy, P.le Tecchio 80, \\ Naples 80125, Italy; E-Mails: angloria@unina.it (A.G.); mariagrazia.raucci@imcb.cnr.it (M.G.R.) \\ * Author to whom correspondence should be addressed; E-Mails: vguarino@unina.it (V.G.); \\ ambrosio@unina.it (L.A.); Tel.: +39-081-242-5929 (L.A.); Fax: +39-081-242-5932 (L.A.).
}

Received: 16 April 2012; in revised form: 19 July 2012 / Accepted: 6 September 2012 /

Published: 14 September 2012

\begin{abstract}
Hydrogels currently represent a powerful solution to promote the regeneration of soft and hard tissues. Primarily, they assure efficient bio-molecular interactions with cells, also regulating their basic functions, guiding the spatially and temporally complex multi-cellular processes of tissue formation, and ultimately facilitating the restoration of structure and function of damaged or dysfunctional tissues. In order to overcome basic drawbacks of traditional synthesized hydrogels, many recent strategies have been implemented to realize multi-component hydrogels based on natural and/or synthetic materials with tailored chemistries and different degradation kinetics. Here, a critical review of main strategies has been proposed based on the use of hydrogels-based devices for the regeneration of complex tissues, i.e., osteo-chondral tissues and intervertebral disc.
\end{abstract}

Keywords: hydrogels; osteochondral tissue; disc nucleus; scaffolds; tissue regeneration

\section{Introduction}

From the beginning, biomaterials have played a key underpinning role in particularizing the properties and functions of medical devices used as prostheses or scaffolds in tissue repair and regenerative medicine. Different approaches based on the use of biodegradable or non degradable polymers have actually been proposed to design novel bioactive systems in turn able (a) to interact with the host tissues; (b) to assist and to improve the healing process; and (c) to replace the functional tissue through the mimicry of morphological characteristics of natural systems. In this context, 
hydrophilic materials and hydrogels, in combination with other material phases strictly organized in composite structures, can be successfully used to satisfy the basic requirements in terms of transport and mechanical properties of natural soft and hard tissue. In this case, the efficacy of the design strategy depends on the mimicking or the supporting action of the proposed engineered platform. Starting from the basic principle of "learning from nature", smart and multifunctional materials can be designed to mimic the behavior of natural soft tissues characterized by complex mechanical loading conditions, mimicking the specific biomechanical and physiological conditions at the interface with the surrounding tissues. In past years, tailor-made hydrogels have demonstrated their ability to guide the tissue growth by bio-molecular interaction with cells or adjacent tissues, thus providing a fine control of their basic functions, guiding the spatially and temporally complex multi-cellular processes of tissue formation and regeneration or facilitating the restoration of structure and function of damaged or dysfunctional tissues [1]. Still, several drawbacks of traditional synthesized hydrogels are concerned with their loss in mechanical properties over time [2] so making their use in load bearing applications often unsatisfactory.

Recently, many recent strategies have focused on composite hydrogels, which afford greater control over each of these aspects, by combining different degradable or non-degradable polymers with tailored chemistries in order to create bioactive systems with customized functional properties $[3,4]$.

So, the need for promoting bio-mimetic integration in the native microenvironment, is opening up a progressive revision of the concept of old hydrogel-based devices, moving towards the design of smart platforms, programmed with peculiar chemical and morphological information able to actively direct the behavior of cells in order to promote tissue regeneration mechanisms.

This paper offers a brief overview of composite hydrogels used largely in biomedical applications and highlights recent advances in material design, focusing attention on different technological approaches for the development of porous scaffolds for tissue engineering and prostheses for tissue replacement.

\section{Hydrogels for Tissue Engineering}

The hydrophilic nature of the natural ECM certainly plays a key role in the basic function of natural tissues. Synthetic hydrogels offer the ability to mimic various distinctive requirements of an ECM-like physicochemical environment and to sustain cellular and tissue function. In the past, they have been largely used to repair and assist regeneration of a variety of tissues, such as cartilage, bone and vasculature $[5,6]$. Now, they have been frequently used as scaffolds for tissue engineering, because they can provide a highly swollen three-dimensional (3D) environment similar to soft tissues and allow diffusion of nutrients and cellular waste through the elastic networks [7,8]. Indeed, hydrogels are hydrophilic crosslinked polymers that are formed by the reaction of one or more monomers, by association of hydrogen bonds or van der Waals interactions between the chains [9]. The crosslinking can be achieved either physically or chemically. While in chemical crosslinking, covalent bonds must be formed, physical crosslinking happens when physical interaction between the chains occurs [10,11]. When hydrogels are in contact with water, they swell and form an insoluble three-dimensional network. Other than injectability, hydrogels display many properties [7] that make them desirable candidates for tissue engineering applications. One of the most important advantages is their aqueous environment, 
which protects cells and sensitive drugs that are incorporated in the network for controlled delivery at the site of injury. The aqueous environment allows transportation of substances, such as nutrients and by-products from cell metabolism, in and out of the hydrogels [8]. Hydrogels can also be derivatized with functional groups that mediate processes such as cell attachment and subsequent spreading [12]. Traditionally, hydrogels were mainly considered for soft tissue regeneration. In the last few years, however, the interest in testing the feasibility of using the beneficial properties of hydrogels for hard tissue regeneration has increased despite the fact that their use is often associated with a number of disadvantages mainly due to their poor tendency to mineralization upon implantation [13].

Even though naturally derived hydrogels have desirable biological properties, they often exhibit degradation profiles that are too fast for hard tissue regeneration [14]. Moreover, chemical characteristics of natural hydrogels such as molecular weight usually display a wide distribution due to their natural origin, which limits the reproducibility and functionality of the materials. On the other hand, synthetic hydrogels can be prepared with tailored and highly reproducible chemical characteristics, thereby enabling the required degradation properties [15]. The combination of the different monomer units results in hydrogels with controlled characteristics in terms of degradation rate, swelling ratios, and mechanical properties [16]. They can be tailored for specific applications with the incorporation of biofunctions, and their transport properties can also be customized by adjusting polymer chain lengths and density [7].

Hydrogels can also be implanted in vivo with minimal invasive techniques. The standard approach consists of seeding a three-dimensional (3D) biomaterial scaffold [17], incorporated with gene vectors, soluble factors and chemical signals to help the new tissue develop during implantation [18]. Furthermore, the crosslinked hydrophilic polymers architecture may provide tissue-like viscoelastic, diffusive transport and interstitial flow characteristics [19]. Naturally-derived and synthetic scaffold materials have been used to exploit the regenerative capacities of host tissues or transplanted cells [20]. Current design and fabrication of organic scaffolds in skeletal tissue engineering (TE) involve a range of materials, such as, protein-based polymers (collagen, fibrin, gelatin, and synthetic polypeptides), natural carbohydrate-based polymers (agarose, alginate, hyaluronate, chitosan, dextran), fully synthetic polymers (polyactive, haluronan and their copolymers with non-degradable polymers: Dacron, Teflon, polyesters, polyurethanes), and composite materials based on the coupling of hydrogels and inorganic compounds.

Although naturally derived biomaterials have proven effective in many basic and clinical applications, the need for custom-made matrices for tissue-specific cell biological investigation drives recapitulation of their key characteristics in synthetic materials. However, their chemical synthesis often causes a reduced control of the material properties because of side reactions coupled with the presence of un-reacted pendant groups and physical bonds (entanglements). Furthermore, gel compositions rapidly degraded in the presence of enzymatic molecules (i.e., hyaluronidase, collagenase) result in a drastic decay of the mechanical performance after implantation, while also presenting slow or delayed response times to external stimuli [21].

The use of synthetic materials has been pursued because the immunogenic and purification issues relating to natural biomaterials are only partially overcome by recombinant protein technologies [1] and the synthetic material properties can be finely controlled and tailored to perform tissue required responses. Although synthetic materials offer these advantages, and are more chemically 
programmable and reproducible, their deficiencies with respect to biological recognition limit their use as tissue regeneration scaffolds [22].

In recent years, hydrogels based on both natural and synthetic polymers have continued to be of interest for the new field of "tissue engineering" for repairing and regenerating a wide variety of tissues and organs [19]. They can reproduce elastic, three-dimensional porous networks able to swell up to $90 \%$ in aqueous solution and to adequately transfer stresses, making them an attractive material for biomedical and tissue engineering applications, such as bone and ligament replacement. Hydrogels typically have lubricating properties, low coefficients of friction and high mechanical strength, very interesting for the regeneration of cartilage and meniscus.

Of note, the addition of synthetic peptides is promising in designing hydrogel carriers with a wealth of bioactive signals programmed directly into the hydrogel matrix. More specifically, it has been verified that incorporation of cell adhesion moieties and biochemical cues allows the promotion of tissue deposition as well as specific enzyme-sensitive sequences which may induce cell-mediated degradation. [23]. For instance, in an effort to recapitulate the native microenvironment that surrounds cells, synthetic extracellular matrix (ECM) hydrogels may be designed by incorporating both proteins and glycosaminoglycans into a single hydrogel matrix. In this configuration, these synthetic ECM analogues can be degraded through hydrolysis of the ester bond associated with the acrylate with a glycosaminoglycans-dependent degradation degree [24]. Degradation properties may be tuned by proper selection of hydrogels. More chemically stable hydrogels can be covalently cross-linked into hydrogel networks by several mechanisms, including chain-growth polymerizations, such as photopolymerization, or step-growth polymerizations, such as Michael-addition reactions. In particular, photopolymerization includes photo-initiated reactions between PEG diacrylate (PEG-DA) molecules or other groups (i.e., thiols) [25]. In this case, functionalized PEGs may be also cross-linked into a biodegradable gel network using peptides that contain protease-sensitive substrate sequences [26]. Alternatively, hydrogels can also be formed by physical or ionic interactions between molecules. This behavior is observed in the case of peptide amphiphiles self-assembly or during the complexation of polymers or polysaccharides with ions [27]. Taking inspiration from nature, hybrid hydrogels have been developed by self-assembling domains, as in silk, with short-chain PEG segments to form hydrogels with good mechanical properties [28]. Moreover, the complexation via the divalent cation calcium is frequently used to obtain hydrogel from alginate or modified alginate polysaccharides [29]. Altering the bulk composition or distribution of mannuronic and guluronic acid units as well as the overall molecular weight of the alginate affects the final hydrogel properties [30].

Interestingly, hydrogels are also largely used as injectable in situ gelling networks and in cell-sheet engineering [31], wound healing and cellular patterning through spray deposition [32,33]. Hydrogels combined with solid particles provide mechanically strong scaffolds largely used for load-bearing applications [34]. To extend the biological performance of synthetic materials, a promising strategy consists of chemically encoding bio-molecular cues (morphogenic bone factors, growth factors, and gene factors) into synthetic platforms [35]. In this context, composite hydrogels represent new frontiers for developing smart materials able to regulate and coordinate events in spatial and temporal modalities guided by biophysical and biochemical signals, as well as biomolecular factors being naturally triggered by the extracellular microenvironment. 


\section{Polymer Based Macroporous Scaffolds for Osteochondral Tissue}

In osteochondral tissue engineering, several three-dimensional cell-based tissue models have been useful in investigating the regeneration processes of cartilage and bone tissue. In this context, the scaffold often represents a critical tool because it is responsible for supporting the medium to deliver cell populations and for inducing the in-growth of surrounding tissue [21]. Firstly, it should guide the tissue growth by bio-molecular interaction with cells or adjacent tissues thus controlling their basic functions, and guiding the spatially and temporally complex multi-cellular processes of tissue formation and regeneration. This is mainly assured by its porous architecture which typically serves as a template that facilitates cell attachment and matrix deposition. In particular, macroporosity within scaffolds is thought to help facilitate cell migration, proliferation, and signalling [36]. Meanwhile, high pore interconnections have implications for facilitated cell migration, abundant cell-cell interaction, and potentially improved vascular growth, thus supporting the intimate cell-cell contact.

In this context, hydrophilic polymers may be efficiently used to build three-dimensional scaffolds that serve as a temporary support for cell growth and new tissue development. The introduction of enhanced pores can provide more space and increased surface area-to-volume ratio of hydrogel scaffolds for cell growth, tissue invasion and local angiogenesis, and facilitate nutrient transport [37]. For example, porous alginate showed enhanced cell proliferation and increased permeability by nearly three orders of magnitude when compared to nonporous conditions [38]. Porous collagen prepared with ice particulates improved cell distribution and chondrogenesis [39]. Moreover, the mechanical and transport properties of porous hydrogels are mainly dictated by pore size and hydrogel density [40]; In particular, pore size is also mandatory for regulating cell behavior, such as neovascularization [41]. More importantly, porosity and pore interconnectivity of the hydrogels have a great impact on the viability of the encapsulated cells by influencing the flow diffusion mechanisms [37]. Therefore, it is crucial to control the various pore features (e.g., pore size, porosity, pore distribution and interconnectivity) within hydrogels. Techniques to control the overall porosity of hydrogels include solvent casting particle leaching, freeze-drying, gas foaming, and electrospinning. Combinations of these methods have been used to fabricate porous hydrogels for many tissue engineering applications [42]. In addition, more advanced control of specific pore features and microarchitecture has been achieved through various micropatterning [43] and micromolding techniques [44]. With these techniques it is possible not only to specifically control individual and group pore architecture, but also to take the next step to create microvascular features to improve integration within host tissues. One of the most studied and widely applied hydrogels is poly(ethylene glycol) (PEG) which has been extensively explored as cell scaffolds [45] as well as in drug delivery devices [46]. Indeed PEG hydrogels recently approved by the FDA for cosmetics, personal care products and pharmaceuticals, show peculiar properties, such as good biocompatibility, non-immunogenity, and resistance to protein adsorption which make their use satisfactory for different biomedical applications including surface modification, bio-conjugation, drug delivery and tissue engineering [47,48]. Three major crosslinking methods have been used to make PEG hydrogels, including radiation of linear or branched PEG polymers [49,50], free radical polymerization (FRP) of PEG acrylates [51], and specific chemical reactions, such as condensation [52], Michael-type additions [53,54], Click chemistry [55], and enzymatic reactions [56]. The most common approach to make PEG hydrogels is photo-polymerization, 
which utilizes light to convert liquid PEG macromer solutions into solid hydrogels at physiological temperature and $\mathrm{pH}$. This method is advantageous for fabricating hydrogel scaffolds in situ with spatial and temporal control and in a variety of $3 \mathrm{D}$ structures with encapsulation of cells and biological agents [57].

PEG acrylates are the major type of macromers used for photo-polymerization, including PEG diacrylate (PEGDA), PEG dimethacrylate (PEGDMA). PEG dimethacrylate (PEGDMA) is an unsaturated linear polyether with methacrylate double bonds that can be crosslinked in situ. Cross-linked PEGDMA, which has been successfully used by several groups both in vitro and in vivo as scaffold material, has been shown to be biocompatible with the unreacted dimethacrylates having relatively low cytotoxicity [58]. For bone tissue engineering purposes, PEG is notable for its lack of protein adsorption and cell adhesion, which play an important role in facilitating osteoblasts matrix deposition during bone remodeling [59,60]. It seems that PEGDMA formation may be too exothermic, and harm the surrounding cells during the process of gelation. However, for tissue engineering purposes, the reactions of PEGDMA hydrogels may be too exothermic, particularly during radical polymerization, which will potentially harm surrounding cells during the process of gelation [61]. Usually, heat release in the crosslinking process of polymers can be decreased by adding inert reagents, which can mediate between the polymer chains and weaken their interactions. PEG hydrogels are attractive scaffolds for providing 3D templates in aqueous environments for tissue regeneration; however, PEG hydrogels typically exhibit minimal or no intrinsic biological activity due to the non adhesive nature of PEG chains [47]. It has been noted that anchorage-dependent cells encapsulated in PEG hydrogels show low viability due to the bio-inert characteristic of PEG [62]. Inspired by nature, researchers have developed a variety of bioactive modified PEG hydrogels to mimic the natural extracellular matrix (ECM) [63].

A valid alternative to the traditional composite systems is also represented by composite scaffolds from the combination of hydrophobic and/or hydrophilic polymers. Recently, PEG has been successfully blended with hydrophobic PCL to form a scaffold with improved molecular transport capability and great potential for grafting bone morphogenic proteins and growth factors [64]. The use of conventional techniques like phase inversion and salt leaching even assures the formation of an interconnected pore network with pre-ordered pore size scale. The preliminary photo-polymerization by ultra-violet (UV) radiation of the composite prevents the undesired removal of PEG, during the ionic dissolution of sodium chloride particles (Figure 1).

Figure 1. Macroporous scaffolds of poly(ethylene glycol) diacrylate (PEGDA) with high swelling properties for osteochondral defect.
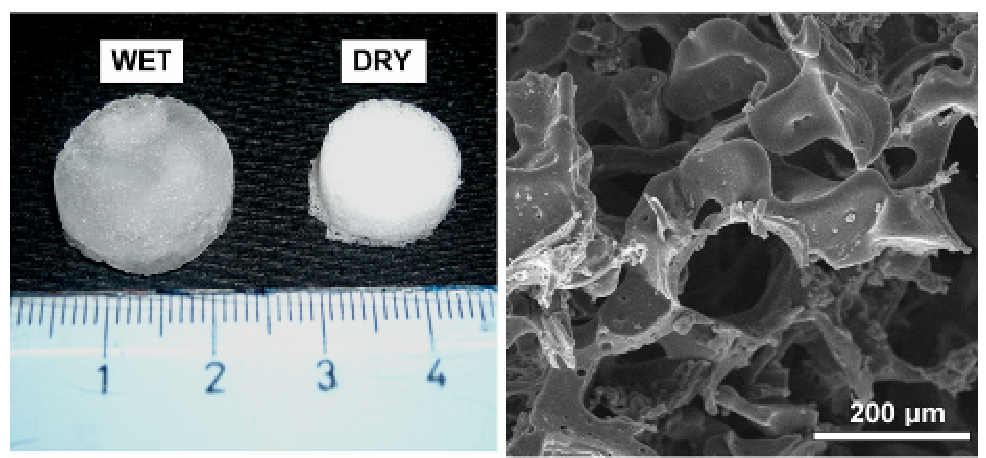
Of note, on the basis of the specific interconnectivity and broad size distribution of the pores, the fluid flow within the hydrogel network may be changed, also affecting the mechanical behavior and permeability of the tissue substitute. Mainly, the integration of hydrogels such as PEG may enable the achievement of some properties similar to highly hydrated tissues in terms of fluid transport properties. Meanwhile, the mechanical integrity may be controlled by crosslinking of the polymeric chain [65]. From this point of view, the hydrophilic properties of the photo-polymerized PEG-based scaffolds allows the retention of high water content - about 10 fold higher than for non photo-polymerized materials. These hydrogels can be efficiently used to encode morphological and functional properties (i.e., porosity, mechanical properties) through the synergic control of the chemical composition and preparation process parameters in order to mimic the behavior of the mature cartilage. In particular, the modulation of the UV exposure time enables the control of water uptake also modulating the transport properties of the substrate, guided by the highly complex chemical composition and structural organization. In order to prevent some of the limitations of non-degradable polymers for clinical uses including acute inflammatory phenomena evidenced by "in vivo" experiments [66], hyaluronan derivates such as HYAFF formulations obtained by chemical modification of purified hyaluronan may be used [67]. The chemical modification of HYAFF consisting of the partial or total esterification of the carboxyl groups of hyaluronic acid, may be controlled by selection of the chemical agents and the esterification extent with considerable effects on the composite biological properties either favoring or, conversely, inhibiting the adhesion of certain cell types [68]. Moreover, by changing the type of ester group introduced or the extent of the esterification, a broad variety of hyaluronan based polymers (HYAFF 11, HYAFF 7) can be subsequently generated and easily processed to produce membranes, fibers, sponges, microspheres and other devices through different techniques (i.e., extrusion, lyophilization or spray drying). Campoccia et al. [69] have demonstrated that benzyl ester Hyaff-11 underwent a spontaneous hydrolytic degradation of the ester bonds even in the absence of any enzymatic activity with complete dissolution after only one week. More recently, biological studies confirmed the relevant effect of esterification degree on the degradation mechanisms as well as on the hydrophilic behavior of the final material, in the case of three dimensional porous scaffolds prepared by freeze drying and salt leaching [70]. In this case, porous architecture is characterized by a well-interconnected and spatially well-distributed macroporosity ranging from 100 to $200 \mu \mathrm{m}$ obtained by the removal of sodium chloride crystals, acting as porogen agent, which allows the control of the pores. The total porosity is equal to $92 \%-93 \%$, consistently with a theoretical value of sodium chloride volume fraction (equal to $91 \%$ ). The hydrogel-like behavior easily determines some of the limitations in the control of shape and pore spatial distribution directly due to the volumetric shrinkage of the HYAFF 11 which occurs during the preparation process when the water content previously adsorbed during the salt leaching step is lost. Hence, the volumetric shrinkage of the hydrogel determines a partial collapse of the polymeric skeleton which explains the "microstructural disorder". Their use combined with aliphatic polyesters such as PCL [67], PLA and PLGA, can allow the stabilization of the pore architecture by the sage combination of hydrophilic and hydrophobic phases ratios without any significant effect on the inflammatory response during the degradation process [70]. This is related to the transport properties of the scaffolds ascribable to the Hyaff gel-like behavior which mimic the native hyaluronan composing the extracellular matrix. 


\section{Composite Hydrogels with Mineral Phases for Hard Tissue Regeneration}

The capacity of a specific class of bone-substituting materials to induce calcification is often referred to as bioactivity, which implies that these materials possess the capacity to promote nucleation and subsequent proliferation of calcium phosphate crystals. Generally, most polymeric materials do not possess this capacity, but the addition of a ceramic phase can still render the resulting composites bioactive by providing nucleation sites for the promotion of hydroxyapatite (HAp) precipitation. The idea of combining an inorganic phase into hydrogels matrices was inspired by the composite nature of bone itself. One of the many advantages of adding an inorganic phase is that the dispersed mineral provides nucleation sites for HAp formation as well as cell adhesion sites that enable integration with surrounding bone tissue [71]. Further, degradation of the temporary hydrogel implant will permit replacement by new bone formation, thus increasing mechanical stability. Degradation times and mechanical properties of organic-inorganic composite materials can be controlled to a large extent by the addition of inorganic phases [72,73]. Moreover, the handling characteristics of such composite materials can be greatly improved, since brittle ceramic particles can be delivered in moldable or even injectable formulations using the elasticity of the hydrogels. Finally [74], the addition of carbonated apatites in polymers can have a neutralizing effect on the acidic $\mathrm{pH}$ caused by the degradation by-products, thus minimizing excessive inflammation around the implantation site. The most commonly used inorganic phases are calcium phosphates and bioglasses. Many calcium phosphate ceramics can be found in the literature with the most representative being $\beta$-tricalcium phosphate $(\beta$-TCP), amorphous calcium phosphate, and HA. This group of ceramics shows strong resemblance to the mineral phase of bone and it is found in many normal or pathological calcified sites in the human body $[75,76]$. Among them, HAp, an important bioceramic found in living vertebrates, was chosen not only for its inertness of chemical reaction but also for its excellent tissue compatibility and osteoconduction. Hydroxyapatite nanoparticles are found to be non-immunogenic and interact with the natural tissue without eliciting significant inflammatory responses [77].

A variety of nanocomposites have been fabricated from polymers and HAp [78,79]. In particular, bioactive inorganic materials may promote a strong bond with the scaffold surface by precipitation of an intermediate layer of HAp, so mineralizing the polymer matrix [75]. In recent years, many studies have been performed on HAp/polymer composite materials prepared from collagen/hydroxyapatite mixtures which are the more interesting candidates to mimic the native composition of bone in repair and tissue engineering strategies. In particular, HAp entrapped in PEGDMA matrices was expected to produce a successful biomaterial for tissue engineering by taking advantage of the PEG's biocompatibility, in situ crosslinking capacity due to the presence of methacrylated groups and the HAp's bioaffinity. In order to achieve a fine dispersion of nanoparticles throughout the polymer preventing nanoparticle aggregation, sol-gel approaches have attracted much attention recently because of their well known advantages, which include homogeneous molecular mixing, low processing temperature and the ability to generate nano-sized inorganic particles dispersed in the polymeric matrix [80]. The development of high-performance nanocomposite hydrogels as synthetic mimics for tissue engineering requires the control of interactions between nanoparticles and the polymer molecules. By combining the polymer characteristics of PEG hydrogels with those of hydroxyapatite nanoparticles (nHAp), elastomeric hydrogels can be synthesized that have unique 
mechanical properties with regard to elongation, compressibility, and toughness. The flexible and elastomeric nature of the nanocomposite networks can be partially attributed to the sufficiently long and flexible PEG chains between cross-linking points and also to some of the physical interactions between polymer and nanoparticles. These nanoparticles also influence cell motility, as evident by the formation of elongated lamellipodia and pseudopodia. Similar results were observed by Chang et al. [81] who studied PEG-silicate nanocomposites and by Gaharwar et al. [82] who showed that the addition of silicate nanoparticles resulted in enhanced cell spreading and proliferation of preosteoblast cells. Several other studies showed that hydroxyapatite promotes adhesion of osteoblast cells. For example, Song et al. [83] fabricated elastomeric nanocomposites composed of poly(2-hydroxyethyl methacrylate) and high amounts of nHAp. The osteoblastic differentiation of the bone marrow stromal cells on these materials was observed to be significantly influenced by the addition of nHAp.

Hydrogels can be also mineralized by biomimetic treatments taking inspiration from the biomineralization process by which native apatite nanocrystals are formed in vivo. Several features of this biomineralization process have been studied for their potential as use in hydrogel mineralization, including alternative soaking treatments in fluids that are saturated with respect to apatite deposition. To produce the apatite coating, scaffold substrates are submitted to a bio-inspired procedure, namely biomimetic treatment, influenced by the method described by Kokubo and co-workers $[84,85]$.

In particular, the treatment combines the preliminary use of a supersaturated SBF solution $(1.5 \times \mathrm{SBF})$ to stimulate the nuclei formation, while a fresh chemically-modified solution $(1 \times \mathrm{SBF})$ is further used, in order to promote the growing of apatite nuclei, once formed.Biomimetic treatment consists of two steps in a $\mathrm{pH}$-controlled environment: during the first step, samples with pre-ordered size were soaked in $1.5 \times \mathrm{SBF}$ where the volume is calculated with respect to the total scaffold material surface by using an exposed surface to SBF volume ratio equal to $10 \mathrm{~mm}^{2} / \mathrm{mL}$, as reported in the literature [86]. The solution temperature was fixed at $37{ }^{\circ} \mathrm{C}$ during the treatment. After the sequential immersion in $1.5 \times \mathrm{SBF}$ (7 days) and in $1 \times \mathrm{SBF}$ (14 and 21 days), all scaffolds were gently rinsed in distilled water to remove excess ions and, then, dried overnight under a laminar hood. The presence of hydroxyapatite on the material surface has the advantage of being able to bind directly to natural bone because of its chemical structure similarity [87,88].

Meanwhile, HA promotes the adsorption of many proteins and other macromolecules and leads to a biological layer that favors cell attachment and osteogenic differentiation [89].

\section{Intervertebral Disc: From Repair to Regeneration}

The intervertebral disc plays a crucial role in the biomechanics of the spine since it mechanically functions as a shock absorber and distributes loads. The intervertebral disc provides flexibility to the spine also enabling the body to twist and bend into a wide range of postures [90]. The natural IVD consists of three different tissues: annulus fibrosus, nucleus pulposus, and endplates. The nucleus is a semi-fluid mass that mainly consists of water and proteoglycans forming a gel-like matrix [91]. It is enclosed within the annulus and the two endplates.

The annulus presents a multi-layered structure consisting of layers characterized by well organized collagen fibers embedded in a proteoglycan-water gel. In adjacent layers the collagen fibers run in 
opposite directions and their orientation varies from $62^{\circ}$ to $45^{\circ}$ with respect to the spine axis, from the edge of the disc inwards to the nucleus [92,93]. The cartilage endplates provide minimal contributions to the overall mechanical behavior of the disc, however, their micropores play an important role in transporting nutrients into the disc [94]. IVD degeneration represents a spinal disease. Even though discectomy and fusion are the two most common surgical approaches for treating a degenerated disc, they are far from ideal treatments since they alter the biomechanics of the spine [90,94-99]. For this reason, the ideal solution to a degenerated disc would involve an artificial disc [90,91,94,97-100].

However, because of the complex structure and function of IVD there have been many difficulties in designing total IVD prostheses able to reproduce the properties of the natural structure, while providing the required durability. It has been well reported that the intervertebral disc prostheses currently available on the market undergo failure mainly because of wear or mismatch between the mechanical proprieties of the implanted device and the natural tissue [99,101]. In addition, current disc prostheses basically consist of a polymeric core interposed between two metallic plates (i.e., LINK SB Charite and ProDisc) possessing standard sizes [99-103].

Soft biological tissues show high strength, flexibility and toughness, and materials with a single structural arrangement do not allow combination of all these features [90,99,100]. Accordingly, a biomimetic approach has been adopted to reproduce the structure of the natural disc involving the use of hydrogels, in order to overcome the drawbacks related to the use of current prostheses.

Initially, poly(2-hydroxyethylmethacrylate) (PHEMA) hydrogels were considered in designing innovative disc prostheses because of their biocompatibility, high permeability and high hydrophilicity [7,99,104,105]. However, it is well documented that in the swollen state the mechanical performances of these materials are not appropriate for load-bearing biomedical applications [99,104,106,107]. Accordingly, their mechanical properties have been enhanced by incorporating a hydrophobic component, such as poly(caprolactone) (PCL), and polymeric fibers [106-109].

For this reason, fiber-reinforced hydrogels able to match the performances of the natural disc and those of the surrounding tissues were manufactured by filament winding and molding technologies, and their mechanical behavior was properly analyzed [7,90,101,107].

For example, an annulus/nucleus substitute made up of a PHEMA/poly(methylmethacrylate) (PMMA) semi-interpenetrating polymer network (s-IPN) composite hydrogel reinforced with poly(ethylene-terephthalate) (PET) fibers has been designed (Figure 2) [90,98-100]. In the swollen state the manufactured nucleus/annulus substitute presented a softer and more hydrophilic inner part and a stiffer and less hydrophilic outer fiber-reinforced ring [90,91,98-100,109,110]. Two artificial endplates made of hydroxyapatite-reinforced polyethylene composite (HAPEX ${ }^{\mathrm{TM}}$ ) were also taken into consideration to anchor the device to the vertebral bodies. The mechanical behavior of this multi-component fiber-reinforced PHEMA-based hydrogel/HAPEX ${ }^{\mathrm{TM}}$ device was further analyzed with static and dynamic mechanical tests [100].

Swollen PHEMA-based composite hydrogels reinforced with PET fibers have displayed compressive J-shaped stress-strain curves that are typical of natural discs. The toe region of the stress-strain curve (i.e., the portion of the curve where concavity is upward) evidences a relatively high flexibility of the structure [90,98-100]. In addition, suitable viscoelastic properties, high dimension stability and endurance have been demonstrated through dynamic tests [90,98-100]. In any case, it is 
possible to tailor the hydrophilicity, the static and dynamic mechanical properties of the hydrogel-based composite device by varying the composition of the matrix, the winding angle and the amount of PET fibers. All of this clearly suggests the possibility to optimize the characteristics of the fiber-reinforced hydrogel-based device at several locations along the spinal column. The ability to design customized hydrogel-based composite disc prostheses by integrating image capture techniques and analyses, computer numeric control machining, filament winding, and molding technologies, was also demonstrated [99].

Figure 2. A multicomponent fiber-reinforced, poly(2-hydroxyethylmethacrylate) (PHEMA)-based Hydrogel/hydroxyapatite-reinforced polyethylene composite (HAPEX ${ }^{\mathrm{TM}}$ ) device for customized intervertebral disc substitution.

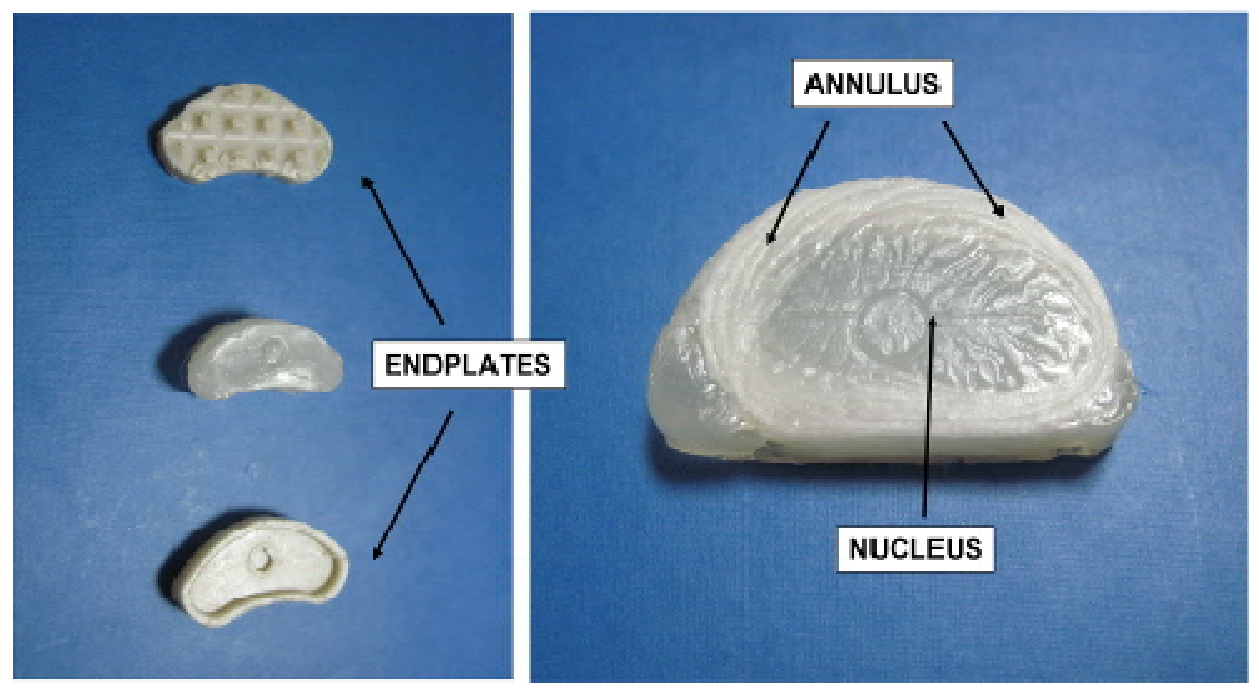

On the other hand, in order to reduce surgical invasiveness the nucleus replacement technique may allow for the restoration of disc height and spine mobility. This approach can be performed if annulus and endplates are still functional and relatively healthy, using a synthetic material or a tissue engineered structure [94,104,111].

In this field, great efforts have been made to design a biocompatible, sterile and safe material as nucleus substitute, especially focusing attention on injectable systems. Different injectable materials based on synthetic in situ curing polymers have been widely studied for the nucleus replacement or augmentation $[94,98,111]$. Although these materials are able to restore disc height and motion, they do not allow the restoration of physiological biomechanics of the spine, also presenting limited biocompatibility and surgical technique limitations [99,111]. However, exothermic reaction, long setting time and surgical approach strongly limit their employment. With the aim of overcoming the above reported limitations, injectable hydrogels have been proposed and studied for nucleus replacement, while also considering their ability to swell and maintain hydrostatic pressure.

In this context, natural and synthetic chemically or physically crosslinked hydrogel-based materials have been taken into account [111-113]. With regard to synthetic hydrogels, physically crosslinked hydrogel based on polyvinyl alcohol (PVA) and polyvinylpyrrolidone (PVP) [114], and on NVP/HEMA ( $N$-vinyl-2-pyrrolidone and 2-hydroxyethylmathacrylate) based hydrogel [115] have been 
studied. In any case, none of the above mentioned materials presented the desired biological cues and the required behavior $[111,116]$.

However, it seems that current clinical practices are unable to properly restore the function of the intervertebral disc. Consequently, in recent years attention has been driven toward disc tissue engineering. Disc tissue engineering represents a quite new field of research and interesting strategies have been proposed in several studies taking into consideration different biomaterials and cell sources [117-119].

In this context, many works have reported progress in the field of disc tissue engineering, highlighting the biomaterial aspect and cellular features [120-123]. The importance of evaluating the mechanical properties of the engineered constructs has been properly evaluated. Even though interesting results have been reported in terms of viable disc-like production, mechanical performances still have to be assessed properly and functional benchmarks have not yet been found [124].

In the field of disc tissue engineering, an initial study was basically focused on defining culture systems in which the phenotypes of nucleus and annulus cells could be preserved. In particular, annulus and nucleus cells were seeded in scaffolds consisting of a crosslinked type I collagen/hyaluronan and the influence of different combinations of growth factors was studied [124]. Following this approach, nucleus and annulus cells have been cultured on a substrate of natural and synthetic polymers, obtaining interesting results [119,123-132].

As for annulus tissue engineering, an interesting approach has been to mimic the peculiar multi-scale structure of the annulus. Trying to reproduce the collagen orientation within a single lamella of the annulus, Shao and Hunter designed a scaffold with aligned alginate/chitosan hybrid fibers that was seeded with annulus cells from a canine intervertebral disc. The obtained results evidenced that cells adhered to the hybrid fibers of the scaffold producing fibrocartilaginous ECM [131].

Some research groups have considered electrospinning as a powerful technique to manufacture scaffolds for annulus tissue engineering [124,133-135]. The basic idea was that the electrospun fibers can closely reproduce the scale of collagen fibers present in most soft tissues, whilst the collection onto a rotating mandrel may provide an aligned mesh consisting of nanofibers. Scaffolds made of aligned electrospun nanofibers and seeded with MSCs were developed and analyzed by Nerurkar et al. (2007, 2008, 2009) and Gruber et al. (2009) [125,127,133,134]. Taking into account the specific organization, the macroscopic mechanical properties of these nanofibrous meshes were suitably evaluated and it was demonstrated that the annulus cells seeded onto aligned nanofibrous scaffolds showing an elongated morphology [134,136,137].

Nerurkar et al. (2009) proposed oriented electrospun PCL scaffolds seeded with MSCs to produce bi-lamellar constructs characterized by opposing collagen orientations [127]. These structures were able to direct the deposition of an organized collagen-rich ECM, thus reproducing the angle-ply multilayered structure of the annulus. Furthermore, uniaxial tensile tests also evidenced that after 10 weeks of in vitro culture these nanofibrous constructs provided a tensile modulus (14.5 $\mathrm{MPa})$ close to the circumferential tensile modulus of the annulus (18.0 MPa). This study demonstrated the possibility of replicating the form and function of the annulus, as well as the role of the opposing fiber orientations in enhancing the tensile performance. 
With regard to nucleus tissue engineering, natural hydrogels have been considered in order to overcome the lack of bioactivity of synthetic hydrogels. In particular, in the field of natural polymers, hyaluronic acid (HA) has attracted researchers' attention, as it has been identified among the glycosaminoglycans present in the nucleus [116]. It is already employed in several biomedical applications (i.e., ophtalmological surgery, orthopaedic fields) as a consequence of its physical, chemical and biological properties [138,139]. However, the fast degradation process of HA and, hence, its short residence time, together with its poor mechanical properties strongly limit its biomedical applications $[111,139]$.

For this reason, different chemical modifications of native hyaluronan have been carried out to obtain chemically and mechanically robust materials, expanding its range of application $[139,140]$. Revell et al. (2007) [141] previously demonstrated that two hyaluronic acid derivatives HYADD3 ${ }^{\circledR}$ and HYAFF $120^{\circledR}$, display properties that could be interesting for tissue engineering applications. HYADD ${ }^{\circledR}$ is a dodecylamide derived from HA that can form physically crosslinked gels in water $[111,142]$, whilst HYAFF $120^{\circledR}$ is a photo-linkable ester obtained by linking the HA molecule with a compound that initiates the polymerization process upon exposure to UV light, consequently forming chemically crosslinked hydrogels. Previous studies showed HYADD3 ${ }^{\circledR}$ was not toxic in cell culture [141] and when loaded with homologous bone marrow stem cells, the cell-loaded HYADD3 ${ }^{\circledR}$ systems provided interesting results in the field of disc tissue engineering [142]. Accordingly, after six weeks from injection into porcine discs, evidence of nucleus regeneration was found.

In contrast to cell-loaded HYADD ${ }^{\circledR}$ systems, HYAFF $120^{\circledR}$ provided successful repair of discs when employed as an injectable acellular material. As demonstrated by histological analyses, a large amount of cellular tissue with chondrocytes able to produce matrix resulted evidently in the center of the disc. Rheological analyses were also performed on HYADD ${ }^{\circledR}$ and HYAFF120 ${ }^{\circledR}$ solutions in order to support the above mentioned positive biological results. As for the viscoelastic properties, storage modulus and loss modulus were evaluated over a wide range of frequencies, evidencing the ability of these materials to reproduce the gel-like behavior of the nucleus. Furthermore, the injection through clinical catheters did not affect their rheological behavior [111,139].

Although some strategies have been proposed for the annulus and the nucleus, many efforts have been made to engineer the entire intervertebral disc. Considering the different structure and composition of the annulus and nucleus, it is easy to understand how disc tissue engineering represents a great challenge that involves a suitable combination of biomaterials and cell sources, as well as chemical and mechanical features.

For example, Mizuno et al. (2004) tried to replicate the anatomic shape of the disc and to engineer both the annulus and nucleus tissues, considering a cell-loaded annulus/nucleus scaffold. It consisted of a mesh of polyglycolic acid coated with polylactic acid as annulus scaffold, seeded with cells from sheep annulus, and of an inner core made of a hydrogel, based on sodium alginate mixed with calcium sulphate seeded with cells from sheep nucleus [143]. Biochemical and biomechanical analyses performed on these tissue-engineered intervertebral discs have provided interesting results [128]. 


\section{Conclusions and Future Perspectives}

Novel approaches in hydrogel and composite design have been proposed for revitalizing the research on new functional biomaterials with the basic idea to jointly improve functional properties and morphological features. The high number of research groups working in the field of hydrogel development and characterization clearly illustrates that hydrogels are ideal candidate materials to be applied in the field of tissue engineering. In the future, materials that better mimic the natural extracellular matrix in terms of composition, structural characteristics, and mechanical properties will be developed. We anticipate that the ideal tissue engineering scaffold will combine the mechanical tailoring possibilities of synthetic polymers with the biomimetic properties of natural materials. In order to mimic the extracellular matrix to a great extent, various solutions exist including natural i.e., glycosaminoglycans, collagen and elastin, semisynthetic, i.e., haluronan derivates, or synthetic polymers such as PEGs, PVA or PVP. In this context, the use of crosslinking gels, semi-interpenetrating polymer networks, and micro or nano-composite hydrogels represent some powerful solutions to improve the biological response of hydrogels. The adaptation of process technologies, traditionally used in scaffold design and composite technologies, to hydrogel science, also enables peculiar transport properties, typical of swollen materials, to be imparted to macroporous scaffolds, thus providing the development of porous matrices with tailored pore morphology and controlled delivery of molecular signals and drugs. However, an optimal scaffold architecture undoubtedly requires further improvements, i.e., by combining the microstructure with the control of surface roughness at the sub micrometric scale. At present, a large number of devices already exist in realizing only one of the above-mentioned prerequisites. Many emerging process techniques such as stereolithography or electrospinning are currently demonstrating their ability to satisfy this major, even essential, challenge by the development of multifunctional devices with a fine tuning of all morphological parameters both on the macroscale as well as on a micro- and nanolevel. Meanwhile, naturally derived polymers (i.e., hyaluronan derivates) have confirmed their potential use in disc regeneration when used in the form of injectable systems with or without cell encapsulation by reproducing the gel-like behavior of the natural nucleus of the intervertebral disc. However, lack of reproducibility and certain risks related to the use of materials from a natural source will impose on scientists the need to investigate new material solutions, i.e., synthesis of macromolecules inspired by nature, such as recombinant proteins, in order to open new routes in medicine and clinical surgery

\section{Acknowledgments}

This study was supported from the Ministero dell'Universita' e della Ricerca by funds of Rete Nazionale di Ricerca TISSUENET N. RBPR05RSM2, MERIT N. RBNE08HM7T, IP STEPS EC FP6500465, "Novel Intervertebral Disc Prostheses", Contract Number G5RD1-CT-2000-00267 and Disc Regeneration project, NMP3-LA-2008-213904.

\section{References}

1. Lutolf, M.P.; Hubbell, J.A.; Synthetic biomaterials as instructive extracellular microenvironments for morphogenesis in tissue engineering. Nat. Biotechnol. 2005, 23, 47-55. 
2. Kopecek, J.; Yang, J. Hydrogels as smart materials. Polym. Int. 2007, 56, 1078-1098.

3. Zustiak, S.P.; Leach, J.B. Hydrolytically degradable poly(ethylene glycol) hydrogel scaffolds with tunable degradation and mechanical properties. Biomacromolecules 2010, 11, 1348-1357.

4. Nie, T.; Baldwin, A.; Yamaguchi, N.; Kiick, K.L. Production of heparin-functionalized hydrogels for the development of responsive and controlled growth factor delivery systems. J. Control Release 2007, 122, 287-296.

5. Buxton, A.N.; Zhu, J.; Marchant, R.E.; West, J.L.; Yoo, J.U.; Johnstone, B. Design and characterization of poly(ethylene glycol) photopolymerizable semi-interpenetrating networks for chondrogenesis of human mesenchymal stem cells. Tissue Eng. 2007, 13, 2549-2560.

6. Hahn, M.S.; McHale, M.K.; Wang, E.; Schmedlen, R.H.; West, J.L. Physiologic pulsatile flow bioreactor conditioning of poly(ethyleneglycol)-based tissue engineered vascular grafts. Ann. Biomed. Eng. 2007, 35, 190-200.

7. Hoffman, A.S. Hydrogels for biomedical applications. Adv. Drug Deliv. Rev. 2002, 54, 3-12.

8. Lee, K.Y.; Mooney, D.J. Hydrogels for tissue engineering. Chem. Rev. 2001, 101, 1869-1879.

9. Peppas, N. Hydrogels. In Biomaterials Science: An Introduction to Materials in Medicine; Ratner, B.D., Hoffman, A.S., Schoen, F.J., Lemons, J.E., Eds.; Elsevier Academic Press: Amsterdam, The Netherlands, 2004; pp. 100-107.

10. Hillel, A.; Shah, P.; Elisseeff, J. Hydrogels in cell encapsulation and tissue engineering. In Biomedical Polymers; Jenkins, M., Ed.; Woodhead Publishing Ltd.: Cambridge, UK, 2007; pp. 57-75.

11. Hennink, W.E.; van Nostrum, C.F. Novel crosslinking methods to design hydrogels. Adv. Drug Deliv. Rev. 2002, 54, 13-36.

12. Kretlow, J.D.; Klouda, L.; Mikos, A.G. Injectable matrices and scaffolds for drug delivery in tissue engineering. Adv. Drug Deliv. Rev. 2007, 59, 263-273.

13. Mano, J.F.; Sousa, R.A.; Boesel, L.F.; Neves, N.M.; Reis, R.L. Bioinert, biodegradable and injectable polymeric matrix composites for hard tissue replacement: State of the art and recent developments. Compos. Sci. Technol. 2004, 64, 789-817.

14. Kim, B.S.; Mooney, D.J. Development of biocompatible synthetic extracellular matrices for tissue engineering. Trends Biotechnol. 1998, 16, 224-230.

15. Nair, L.S.; Laurencin, C.T. Biodegradable polymers as biomaterials. Prog. Polym. Sci. 2007, 32, $762-798$.

16. Tessmar, J.K.; Gopferich, A.M. Customized PEG-derived copolymers for tissue-engineering applications. Macromol. Biosci. 2007, 7, 23-39.

17. Mooney, D.J.; Mikos, A.G. Growing new organs. Sci. Am. 1999, 280, 60-65.

18. Lum, L.; Elisseeff, J. Injectable hydrogels for cartilage tissue engineering. In Topics in Tissue Engineering; Ashammakhi, N., Ferretti, P., Eds.; University of Oulu: Oulu, Finland, 2003; pp. 1-25.

19. Woerly, S. Porous hydrogels for neural tissue engineering. Porous Mater. Tissue Eng. 1997, 250, 53-68.

20. Griffith, L.G.; Naughton, G. Tissue engineering-Current challenges and expanding opportunities. Science 2002, 295, 1009-1014. 
21. Matthew, H.W.; Salley, S.O.; Peterson, W.D.; Klein, M.D. Complex coacervate microcapsules for mammalian cell culture and artificial organ development. Biotechnol. Prog. 1993, 9, 510-519.

22. Guarino, V.; Causa, F.; Ambrosio, L. Bioactive scaffolds for bone and ligament tissue. Exp. Rev. Med. Dev. 2007, 4, 406-418.

23. Kisiday, J.; Jin, M.; Kurz, B.; Hung, H.; Semino, C.; Zhang, S.; Grodzinsky, A.J. Self-assembling peptide hydrogel fosters chondrocyte extracellular matrix production and cell division: Implications for cartilage tissue repair. Proc. Nat. Acad. Sci. USA 2002, 99, 9996-10010.

24. Shu, X.Z.; Ahmad, S.; Liu, Y.C.; Prestwich, G.D. Synthesis and evaluation of injectable, in situ crosslinkable synthetic extracellular matrices for tissue engineering. J. Biomed. Mater. Res. A 2006, 79A, 902-912.

25. Lutolf, M.P.; Hubbell, J.A. Synthesis and physicochemical characterization of end-linked poly(ethylene glycol)-co-peptide hydrogels formed by michael-type addition. Biomacromolecules 2003, 4, 713-722.

26. Lévesque, S.G.; Shoichet, M.S. Synthesis of enzyme-degradable, peptide-cross-linked dextran hydrogels. Bioconjugate Chem. 2007, 18, 874-885.

27. Patterson, J.; Martino, M.M.; Hubbell, J.A. Biomimetic materials in tissue engineering. Mater. Today 2010, 13, 14-22.

28. Rathore, O.; Sogah, D.Y. Synthesis of triblock copolymers with poly(Ala). J. Am. Chem. Soc. 2001, 123, 5231-5239.

29. Freeman, I.; Cohen, S. The influence of the sequential delivery of angiogenic factors from affinity-binding alginate scaffolds on vascularisation. Biomaterials 2009, 30, 2122-2131.

30. Sikorski, P.; Mo, F.; Skjaek-Braek, G.; Stokke, B.T. Evidence for egg-box-compatible interactions in calcium-alginate gels from fiber X-ray diffraction. Biomacromolecules 2007, 8, 2098-2103.

31. Kikuchi, A.; Okano, T. Nanostructured designs of biomedical materials: applications of cell sheet engineering to functional regenerative tissues and organs. J. Control Release 2005, 101, 69-84.

32. Roberts, A.; Wyslouzil, B.E.; Bonassar, L. Aerosol delivery of mammalian cells for tissue engineering. Biotechnol. Bioeng. 2005, 91, 801-807.

33. Nahmias, Y.; Arneja, A.; Tower, T.T.; Renn, M.J.; Odde, D.J. Cell patterning on biological gels via cell spraying through a mask. Tissue Eng. 2005, 11, 701-708.

34. Spitzer, R.S.; Perka, C.; Lindenhayn, K.; Zippel, H. Matrix engineering for osteogenic differentiation of rabbit periosteal cells using alpha-tricalcium phosphate particles in a three-dimensional fibrin culture. J. Biomed. Mater. Res. 2002, 59, 690-696.

35. Fedorovich, N.E.; Alblas, J.; de Wijn, J.R.; Hennink, W.E.; Verbout, A.J.; Dhert, W.J.A. Hydrogels as extracellular matrices for skeletal tissue engineering: state-of-the-art and novel application in organ printing. Tissue Eng. 2007, 13, 1905-1925.

36. Yoon, D.M.; Fisher, J.P. Chondrocyte signaling and artificial matrices for articular cartilage engineering. Adv. Exp. Med. Biol. 2006, 585, 67-86.

37. Hollister, S.J. Porous scaffold design for tissue engineering. Nat. Mater. 2005, 4, 518-524. 
38. Hwang, C.M.; Sant, S.; Masaeli, M.; Kachouie, N.N.; Zamanian, B.; Lee, S.-H.; Khademhosseini, A. Fabrication of three-dimensional porous cell-laden hydrogel for tissue engineering. Biofabrication 2010, doi:10.1088/1758-5082/2/3/035003.

39. Lu, H.; Ko, Y.G.; Kawazoe, N.; Chen, G. Cartilage tissue engineering using funnel-like collagen sponges prepared with embossing ice particulate templates. Biomaterials 2010, 31, 5825-5835.

40. Sopyan, I.S.; Khalid, K.A. Porous hydroxyapatite for artificial bone applications. Sci. Technol. Adv. Mater. 2007, 8, 116-123.

41. Druecke, D.; Pieper, J.; Ugarkovic, M.; Steinau, H.U.; Homann, H.H. Neovascularization of poly(ether ester) block-copolymer scaffolds in vivo: Long-term investigations using intravital fluorescent microscopy. J. Biomed. Mater. Res. A 2004, 68, 10-18.

42. Khademhosseini, A.; Bettinger, C.; Karp, J.M.; Yeh, J.; Ling, Y.; Borenstein, J.; Fukuda, J.; Langer, R. Interplay of biomaterials and micro-scale technologies for advancing biomedical applications. J. Biomater. Sci. Polym. Ed. 2006, 17, 1221-1240.

43. Du, Y.; Lo, E.; Ali, S.; Khademhosseini, A. Directed assembly of cell-laden microgels for fabrication of 3D tissue constructs. Proc. Nat. Acad. Sci. USA 2008, 105, 9522-9527.

44. Yeh, J.; Ling, Y.; Karp, J.M.; Gantz, J.; Chandawarkar, A.; Eng, G.; Blumling, J.; Langer, R.; Khademhosseini, A. Micromolding of shape-controlled, harvestable cellladen hydrogels. Biomaterials 2006, 27, 5391-5398.

45. Krsko, P.; Libera, M. Biointeractive hydrogels. Mater. Today 2005, 8, 36-44.

46. Bell, C.L.; Peppas, N.A. Water, solute and protein diffusion in physiologically responsive hydrogels of poly (methacrylic acid-g-ethylene glycol). Biomaterials 1996, 17, 1203-1218.

47. Alcantar, N.A.; Aydil, E.S.; Israelachvili, J.N. Polyethylene glycol-coated biocompatible surfaces. J. Biomed. Mater. Res. 2000, 51, 343-351.

48. Singh, D.K.; Ray, A.R. Biomedical applications of chitin, chitosan, and their derivatives. J. Macromol. Sci. Rev. Macromol. Chem. Phys. 2000, C40, 69-83.

49. Peppas, N.A.; Keys, K.B.; Torres-Lugo, M.; Lowman, A.M. Poly(ethylene glycol)- containing hydrogels in drug delivery. J. Control Release 1999, 62, 81-87.

50. Keys, K.B.; Andreopoulos, F.M; Peppas, N.A. Poly(ethylene glycol) star polymer hydrogels. Macromolecules 1998, 31, 8149-8156.

51. Beamish, J.A.; Zhu, J.; Kottke-Marchant, K.; Marchant, R.E. The effects of mono- acrylate poly(ethylene glycol) on the properties of poly(ethylene glycol) diacrylate hydrogels used for tissue engineering. J. Biomed. Mater. Res. A 2010, 92, 441-450.

52. Hubbell, J.A. Synthetic biodegradable polymers for tissue engineering and drug delivery. Curr. Opin. Solid State Mater. Sci. 1998, 3, 246-251.

53. Metters, A.; Hubbell, J. Network formation and degradation behavior of hydrogels formed by Michael-type addition reactions. Biomacromolecules 2005, 6, 290-301.

54. Park, Y.; Lutolf, M.P.; Hubbell, J.A.; Hunziker, E.B.; Wong, M. Bovine primary chondrocyte culture in synthetic matrix metalloproteinase-sensitive poly(ethylene glycol)-based hydrogels as a scaffold for cartilage repair. Tissue Eng. 2004, 10, 515-522.

55. Polizzotti, B.D.; Fairbanks, B.D.; Anseth, K.S. Three-dimensional biochemical patterning of Click-based composite hydrogels via thiol-ene photo- polymerization. Biomacromolecules 2008, 9, 1084-1087. 
56. Ehrbar, M.; Rizzi, S.C.; Schoenmakers, R.G.; Miguel, B.S.; Hubbell, J.A.; Weber, F.E. Biomolecular hydrogels formed and degraded via site-specific enzymatic reactions. Biomacromolecules 2007, 8, 3000-3007.

57. Truong, K.; West, J.L. Photopolymerizable hydrogels for tissue engineering applications. Biomaterials 2002, 23, 4307-4314.

58. Lin-Gibson, S.; Bencherif, S.; Cooper, J.A.; Wetzel, S.J.; Antonucci, J.M.; Vogel, B.M. Synthesis and characterization of PEG dimethacrylates and their hydrogels. Biomacromolecules 2004, 5, 1280-1287.

59. Schmedlen, K.H.; Masters, K.S.; West, J.L. Photocrosslinkable polyvinyl alcohol hydrogels that can be modified with cell adhesion peptides for use in tissue engineering. Biomaterials 2002, 23, 4325-4332.

60. Yang, F.; Williams, C.G.; Wang, D.A.; Lee, H.; Manson, P.N.; Elisseeff, J. The effect of incorporating RGD adhesive peptide in polyethylene glycol diacrylate hydrogel on osteogenesis of bone marrow stromal cells. Biomaterials 2005, 26, 5991-5998.

61. Shu, X.Z.; Liu, Y.C.; Palumbo, F.S.; Lu, Y.; Prestwich, G.D. In situ crosslinkable hyaluronan hydrogels for tissue engineering. Biomaterials 2004, 25, 1339-1348.

62. Nuttelman, C.R.; Rice, M.A.; Rydholm, A.E.; Salinas, C.N.; Shah, D.N.; Anseth, K.S.; Macromolecular monomers for the synthesis of hydrogel niches and their application in cell encapsulation and tissue engineering. Prog. Polym. Sci. 2008, 33, 167-170.

63. Lutolf, M.P. Spotlight on hydrogels. Nat. Mater. 2009, 8, 451-453.

64. Guarino, V.; Gloria, A.; de Santis, R.; Ambrosio, L. Composite hydrogels for scaffold design, tissue engineering and prostheses. In Biomedical Applications of Hydrogels Handbook; Ottenbrite, R.M., Park, K., Okano, T., Eds.; Springer: New York, NY, USA, 2010; pp. 227-245.

65. Hui, P.W.; Leung, P.C.; Sher, A. Fluid conductance of cancellous bone graft as a predictor for graft-host interface healing. J. Biomech. 1996, 29, 123-132.

66. Santavirta, S.; Konttinen, Y.T.; Saito, T.; GroKnblad, M.; Partio, E.; Kemppinen, P.; Rokkanen, P. Immune response to polyglycolic acid implants. J. Bone Jt. Surg. Br. 1990, 72, 597-600.

67. Guarino, V.; Lewandowska, M.; Bil, M.; Polak, B.; Ambrosio, L. Morphology and degradation properties of $\mathrm{pcl} /$ hyaffl1-based composite scaffolds with multiscale degradation rate. Compos. Sci. Technol. 2010, 70, 1826-1837.

68. Campoccia, D.; Doherty, P.; Radice, M.; Brun, P.; Abatangelo, G.; Williams, D.F. Semisynthetic resorbable materials from hyaluronan esterification. Biomaterials 1998, 19, 2101-2127.

69. Manferdini, C.; Guarino, V.; Zini, N.; Raucci, M.G.; Ferrari, A.; Grassi, F.; Gabusi, E.; Squarzoni, S.; Facchini, A.; Ambrosio, L.; Lisignoli, G. Mineralization behavior with mesenchymal stromal cells in a biomimetic hyaluronic acid-based scaffold. Biomaterials 2010, 31, 3986-3996.

70. Seal, B.L.; Otero, T.C.; Panitch, A. Polymeric biomaterials for tissue and organ regeneration. Mater. Sci. Eng. 2001, 34, 147-230.

71. Rea, S.M.; Best, S.M.; Bonfield, W. Bioactivity of ceramic-polymer composites with varied composition and surface topography. J. Mater. Sci. Mater. Med. 2004, 15, 997-1005. 
72. Rezwan, K.; Chen, Q.Z.; Blaker, J.J.; Boccaccini, A.R. Biodegradable and bioactive porous polymer/inorganic composite scaffolds for bone tissue engineering. Biomaterials 2006, 27, 3413-3431.

73. Neumann, M.; Epple, M. Composites of calcium phosphate and polymers as bone substitution materials. Eur. J. Trauma 2006, 32, 125-131.

74. Schiller, C.; Epple, M. Carbonated calcium phosphates are suitable pH-stabilising fillers for biodegradable polyesters. Biomaterials 2003, 24, 2037-2043.

75. Dorozhkin, S.V.; Epple, M. Biological and medical significance of calcium phosphates. Angew. Chem. Int. Ed. Engl. 2002, 41, 3130-3146.

76. Balasundaram, G.; Webster, T. A perspective on nanophase materials for orthopedic implant applications. J. Mater. Chem. 2006, 16, 3737-3745.

77. Paul, D.R.; Robeson, L.M. Polymer nanotechnology: Nanocomposites. Polymer 2008, 49, 3187-3204.

78. Rogel, M.R.; Qiu, H.; Ameer, G.A. The role of nanocomposites in bone regeneration. J. Mater. Chem. 2008, 18, 4233-4241.

79. Kamitakahara, M.; Ohtsuki, C.; Miyazaki, T. Review paper: Behavior of ceramic biomaterials derived from tricalcium phosphate in physiological condition. J. Biomater. Appl. 2008, 23, 197-212.

80. Raucci, M.G.; Guarino, V.; Ambrosio, L. Hybrid composite scaffolds prepared by sol-gel method for bone regeneration. Compos. Sci. Technol. 2010, 70, 1861-1868.

81. Chang, C.W.; van Spreeuwel, A.; Zhang, C.; Varghese, S. PEG/clay nanocomposite hydrogel: A mechanically robust tissue engineering scaffold. Soft Matter 2010, 6, 5157-5164.

82. Gaharwar, A.K.; Dammu, S.A.; Canter, J.M.; Wu, C.J.; Schmidt, G. Highly extensible, tough, and elastomeric nanocomposite hydrogels from poly(ethylene glycol) and hydroxyapatite nanoparticles. Biomacromolecules 2011, 12, 1641-1650.

83. Song, J.; Xu, J.; Filion, T.; Saiz, E.; Tomsia, A.P.; Lian, J.B.; Stein, G.S.; Ayers, D.C.; Bertozzi, C.R.J. Elastomeric high-mineral content hydrogel-hydroxyapatite composites for orthopedic applications. Biomed. Mater. Res. Part A 2009, 89A, 1098-1107.

84. Abe, Y.; Kokubo, T.; Yamamuro, T. Apatite coating on ceramics, metals and polymers utilizing a biological process. J. Mater. Sci. Mater. Med. 1990, 1, 233-238.

85. Tanashi, M.; Yao, T.; Kokubo, T.; Minoda, M.; Miyamoto, T.; Nakamura, T.; Yamamuro, T. Apatite coating on organic polymers by biomimetic process. J. Am. Ceram. Soc. 1994, 7, 2805-2808.

86. Tanahashi, M.; Hata, K.; Kokubo, T.; Minoda, M.; Miyamoto, T.; Nakamura, T. Effect of substrate on apatite formation by a biomimetic process. In Bioceramics; Yamamuro, T., Kokubo, T., Nakamura, T., Eds.; Kobunshi-Kankokai, Inc.: Kyoto, Japan, 1992; Volume 5, pp. 57-64.

87. Jao, Y.P.; Liu, Z.H.; Cui, F.Z.; Zhou, C.R. Effect of hydrolysis pretreatment on the formation of bone-like apatite on poly(L-lactide) by mineralization in simulated body fluids. J. Bioact. Compat. Polym. 2007, 22, 492-507. 
88. Ren, Y.J.; Sun, X.D.; Cui, F.Z.; Wei, Y.T.; Cheng, Z.J.; Kong, X.D. Preparation and characterization of antheraea pernyi silk fibroin based nanohydroxyapatite composites. J. Bioact. Compat. Polym. 2007, 22, 465-474.

89. Zhou, D.S.; Zhao, K.B.; Li, Y.; Cui, F.Z.; Lee, I.S. Repair of segmental defects with nano-hydroxyapatite/collagen/PLA composite combined with mesenchymal stem cells. J. Bioact. Compat. Polym. 2006, 21, 373-384.

90. Gloria, A.; de Santis, R.; Causa, F.; Ambrosio, L. Composite materials for spinal implants. In Biomedical Composites; Ambrosio, L., Ed.; Woodhead Publishing Limited, CRC Press: Cambridge, UK, 2010; pp. 178-200.

91. Rothman, R.H.; Simeone, F.A. The Spine, 3rd ed.; WB Saunders Company: Philadelphia, PA, USA, 1992.

92. Markolf, K.L.; Morris, J.M. The structural components of the intervertebral disc. J. Bone Jt. Surg. Am. 1974, 56, 675-687.

93. Cassidy, J.J.; Hiltner, A.; Baer, E. Hierarchical structure of the intervertebral disc. Connect Tissue Res. 1989, 23, 75-88.

94. Bao, Q.B.; McCullen, G.M.; Higham, P.A.; Dumbleton, J.H.; Yuan, H.A. The artificial disc: Theory, design and materials. Biomaterials 1996, 17, 1157-1167.

95. Goel, V.K.; Nishiyama, K.; Weinstein, J.N.; Liu, Y.K. Mechanical properties of lumbar spinal motion segments as affected by partial disc removal. Spine 1986, 11, 1008-1012.

96. Hanley, E.N., Jr.; Shapiro, D.E. The development of low-back pain after excision of a lumber disc. J. Bone Jt. Surg. Am. 1989, 71A, 719-721.

97. Bao, Q.B.; Yuan, H.A. Artificial disc technology. Neurosurg. Focus 2000, 9, 1-7.

98. Gloria, A.; Causa, F.; de Santis, R.; Netti, P.A.; Ambrosio, L. Dynamic-mechanical properties of a novel composite intervertebral disc prosthesis. J. Mater. Sci. Mater. Med. 2007, 18, 2159-2165.

99. Gloria, A.; de Santis, R.; Ambrosio, L.; Causa, F.; Tanner, K.E. A multi-component fiber-reinforced PHEMA-based hydrogel/HAPEX ${ }^{\mathrm{TM}}$ device for customized intervertebral disc prosthesis. J. Biomater. Appl. 2011, 25, 795-810.

100. Gloria, A.; Ronca, D.; Russo, T.; D’Amora, U.; Chierchia, M.; de Santis, R.; Nicolais, L.; Ambrosio, L. Technical features and criteria in designing fiber-reinforced composite materials: from the aerospace and aeronautical field to biomedical applications. J. Appl. Biomater. Biomech. 2011, 9, 151-163.

101. Shikinami, Y.; Kotani, Y.; Cunningham, B.W.; Abumi, K.; Kaneda, K. A biomimetic artificial disc with improved mechanical properties compared to biological intervertebral discs. Adv. Funct. Mater. 2004, 14, 1039-1046.

102. Buttner-Janz, K. The Development of the Artificial Disc SB Charité; Hundley \& Associates Inc.: Dallas, TX, USA, 1992.

103. Traynelis, V.C. Spinal arthroplasty. Neurosurg. Focus 2002, 13, 1-7.

104. Netti, P.A.; Shelton, J.C.; Revell, P.A.; Pirie, C.; Smith, S.; Ambrosio, L.; Nicolais, L.; Bonfield, W. Hydrogels as an interface between bone and an implant. Biomatererials 1993, 14, 1098-1104.

105. Peppas, N.A.; Bures, P.; Leobandung, W.; Ichikawa, H. Hydrogels in pharmaceutical formulations. Eur. J. Pharm. Biopharm. 2000, 50, 27-46. 
106. Ambrosio, L.; Netti, P.A.; Iannace, S.; Huang, S.J.; Nicolais, L. Composite hydrogels for intervertebral disc prostheses. J. Mater. Sci. Mater. Med. 1996, 7, 251-254.

107. Ambrosio, L.; de Santis, R.; Nicolais, L. Composite hydrogels for implants. Proc. Inst. Mech. Eng. H 1998, 212, 93-99.

108. Davis, P.A.; Huang, S.J.; Ambrosio, L.; Nicolais, L.; Ronca, D. A biodegradable composite artificial tendon. J. Mater. Sci. Mater. Med. 1991, 3, 359-364.

109. De Santis, R.; Sarracino, F.; Mollica, F.; Netti, P.A.; Ambrosio, L.; Nicolais, L. Continuous fibre reinforced polymers as connective tissue replacement. Compos. Sci. Technol. 2004, 64, 861-871.

110. Ambrosio, L.; Causa, F.; de Santis, R.; Nicolais, L. Composite Biomimetic Total Intervertebral Disc Prosthesis. WO Patent 2007/007284, 18 January 2007.

111. Gloria, A.; Borzacchiello, A.; Causa, F.; Ambrosio, L. Rheological characterisation of Hyaluronic acid derivatives as injectable materials toward nucleus pulposus regeneration. J. Biomater. Appl. 2012, 26, 745-759.

112. Oka, M.; Noguchi, T.; Kumar, P.; Ikeuchi, K.; Yamamuro, T.; Hyon, S.H.; Ikada, Y. Development of an artificial articular cartilage. Clin. Mater. 1990, 6, 361-381.

113. Gopferich, A. Mechanisms of polymer degradation and erosion. Biomaterials 1996, 17, 103-114.

114. Joshi, A.; Fussell, G.; Thomas, J.; Hsuan, A.; Lowman, A.; Karduna, A.; Vresilovic, E.; Marcolongo, M. Functional compressive mechanics of a PVA/PVP nucleus pulposus replacement. Biomaterials 2006, 27, 176-184.

115. Boelen, E.J.H.; van Hooy-Corstjens, C.S.J.; Bulstra, S.K.; van Ooij, A.; van Rhijn, L.W.; Koole, L.H. Intrinsically radiopaque hydrogels for nucleus pulposus replacement. Biomaterials 2005, 26, 6674-6683.

116. Leone, G.; Torricelli, P.; Chiumiento, A.; Facchini, A.; Barbucci, R. Amidic alginate hydrogel for nucleus pulposus replacement. J. Biomed. Mater. Res. A 2008, 84, 391-401.

117. Butler, W.F. Comparative anatomy and development of the mammalian disc. In The Biology of the Intervertebral Disc; Gosh, P., Ed.; CRC Press: Boca Raton, FL, USA, 1989; pp. 84-108.

118. Nerurkar, N.L.; Elliott, D.M.; Mauck, R.L. Mechanical design criteria for intervertebral disc tissue engineering. J. Biomech. 2010, 43, 1017-1030.

119. Gloria, A.; Russo, T.; de Santis, R.; Ambrosio, L. Nucleus regeneration. In Biomaterials for Spinal Surgery; Ambrosio, L., Tanner, E., Eds.; Woodhead Publishing Limited: Cambridge, UK, 2012; pp. 563-581.

120. O'Halloran, D.M.; Pandit, A.S. Tissue-engineering approach to regenerating the intervertebral disc. Tissue Eng. 2007, 13, 1927-1954.

121. Kandel, R.A.; Roberts, S.; Urban, J. Tissue engineering and the intervertebral disc: The challenges. Eur. Spine J. 2008, 17, 480-491.

122. Alini, M.; Li, W.; Markovic, P.; Aebi, M.; Spiro, R.C.; Roughley, P.J. The potential and limitations of a cell-seeded collagen/hyaluronan scaffold to engineer an intervertebral disc-like matrix. Spine 2003, 28, 446-454.

123. Chang, G.; Kim, H.J.; Kaplan, D.; Vunjak-Novakovic, G.; Kandel, R.A. Porous silk scaffolds can be used for tissue engineering annulus fibrosus. Eur. Spine J. 2007, 16, 1848-1857.

124. Chang, G.; Kim, H.J.; Vunjak-Novakovic, G.; Kaplan, D.; Kandel, R.A. Enhancing annulus fibrosus tissue formation in porous silk scaffolds. J. Biomed. Mater. Res. A 2010, 92, 43-51. 
125. Gruber, H.E.; Hoelscher, G.; Ingram, J.A.; Hanley, E. Culture of human annulus fibrosus cells on polyamide nanofibers: Extracellular matrix production. Spine 2009, 34, 4-9.

126. Mizuno, H.; Roy, A.K.; Zaporojan, V.; Vacanti, C.A.; Ueda, M.; Bonasser, L.J. Biomechanical and biochemical characterization of composite tissue-engineered intervertebral discs. Biomaterials 2009, 27, 362-370.

127. Nerurkar, N.L.; Baker, B.M.; Sen, S.; Wible, E.E.; Elliott, D.M.; Mauck, R.L. Nanofibrous biologic laminates replicate the form and function of the annulus fibrosus. Nat. Mater. 2009, 8 , 986-992.

128. Rong, Y.; Sugumaran, G.; Silbert, J.E.; Spector, M. Proteoglycans synthesized by canine intervertebral disc cells grown in a type Icollagen-glycosaminoglycan matrix. Tissue Eng. 2002, 8, 1037-1047.

129. Sato, M.; Asazuma, T.; Ishihara, M.; Kikuchi, T.; Masuoka, K.; Ichimura, S.; Kikuchi, M.; Kurita, A.; Fujikawa, K. An atelocollagen honeycomb-shaped scaffold with a membrane seal (ACHMS-scaffold) for the culture of annulus fibrosus cells from an intervertebral disc. J. Biomed. Mater. Res. A 2003, 64A, 249-256.

130. Sato, M.; Kikuchi, M.; Ishihara, M.; Asazuma, T.; Kikuchi, T.; Masuoka, K.; Hattori, H.; Fujikawa, K. Tissue engineering of the intervertebral disc with cultured annulus fibrosus cells using atelocollagen honeycomb-shaped scaffold with a membrane seal (ACHMS scaffold). Med. Biol. Eng. Comp. 2003, 41, 365-371.

131. Shao, X.; Hunter, C.J. Developing an alginate/chitosan hybrid fiber scaffold for annulus fibrosus cells. J. Biomed. Mater. Res. A 2007, 82, 701-710.

132. Wan, Y.; Feng, G.; Shen, F.H.; Laurencin, C.T.; Li, X. Biphasic scaffold for annulus fibrosus tissue regeneration. Biomaterials 2008, 29, 643-652.

133. Nerurkar, N.L.; Elliott, D.M.; Mauck, R.L. Mechanics of oriented electrospun nanofibrous scaffolds for annulus fibrosus tissue engineering. J. Orthop Res. 2007, 25, 1018-1028.

134. Nerurkar, N.L.; Mauck, R.L.; Elliott, D.M. ISSLS prize winner: Integrating theoretical and experimental methods for functional tissue engineering of the annulus fibrosus. Spine 2008, 33, 2691-2701.

135. Yang, L.; Kandel, R.A.; Chang, G.; Santerre, J.P. Polar surface chemistry of nanofibrous polyurethane scaffold affects annulus fibrosus cell attachment and early matrix accumulation. J. Biomed. Mater. Res. A 2008, 91, 1089-1099.

136. Courtney, T.; Sacks, M.S.; Stankus, J.; Guan, J.; Wagner, W.R. Design and analysis of tissue engineering scaffolds that mimic soft tissue mechanical anisotropy. Biomaterials 2006, 27, 3631-3638.

137. Li, W.J.; Mauck, R.L.; Cooper, J.A.; Yuan, X.; Tuan, R.S. Engineering controllable anisotropy in electrospun biodegradable nanofibrous scaffolds for musculoskeletal tissue engineering. J. Biomech. 2007, 40, 1686-1693.

138. Gustafson, S. Hyaluronan in drug delivery. In The Chemistry, Biology and Medical Applications of the Hyaluronan and its Derivatives; Laurent, T.C., Balazs, E.A., Eds.; Portland Press: London, UK, 1997; pp. 291-304. 
139. Borzacchiello, A.; Gloria, A.; Ambrosio, L. Spinal disc implants using hydrogels. In Biomedical Hydrogels: Biochemistry, Manufacture and Medical Applications; Rimmer, S., Ed.; Woodhead Publishing Limited: Cambridge, UK, 2011; pp. 103-117.

140. Maltese, A.; Borzacchiello, A.; Mayol, L.; Bucolo, C.; Nicolais, L.; Ambrosio, L. Novel polysaccharides-based viscoelastic formulations for ophthalmic surgery: Rheological characterization. Biomaterials 2006, 27, 5134-5142.

141. Revell, P.A.; Damien, E.; Di Silvio, L.; Gurav, N.; Longinotti, C.; Ambrosio, L. Tissue engineered intervertebral disc repair in the pig using injectable polymers. J. Mater. Sci. Mater. Med. 2007, 18, 303-308.

142. Borzacchiello, A.; Mayol, L.; Schiavinato, A.; Ambrosio, L. Effect of hyaluronic acid amide derivative on equine synovial fluid viscoelasticity. J. Biomed. Mater. Res. A 2010, 92, 1162-70.

143. Mizuno, H.; Roy, A.K.; Vacanti, C.A.; Kojima, K.; Ueda, M.; Bonassar, L.J. Tissue-engineered composites of annulus fibrosus and nucleus pulposus for intervertebral disc replacement. Spine 2004, 29, 1290-1297.

(C) 2012 by the authors; licensee MDPI, Basel, Switzerland. This article is an open access article distributed under the terms and conditions of the Creative Commons Attribution license (http://creativecommons.org/licenses/by/3.0/). 